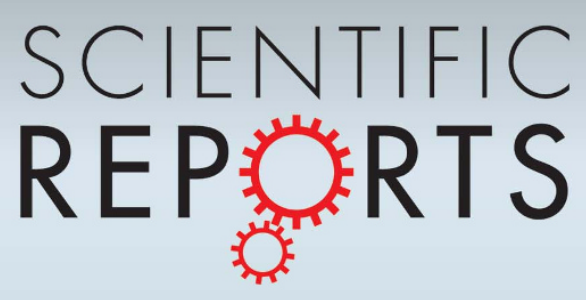

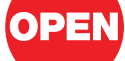

SUBJECT AREAS:

SENSORY SYSTEMS

MOLECULAR NEUROSCIENCE

PLASTICITY

NEURODEGENERATION

Received

15 December 2011

Accepted

5 January 2012

Published

20 January 2012

Correspondence and requests for materials should be addressed to

M.W.A. lalbers. mark@mgh.harvard.

edu)

\title{
The precision of axon targeting of mouse olfactory sensory neurons requires the BACE 1 protease
}

\author{
Luxiang Cao, Gregory T. Rickenbacher, Steve Rodriguez, Thomas W. Moulia \& Mark W. Albers
}

MassGeneral Institute of Neurodegenerative Disease, Dept. of Neurology, Harvard Medical School, Boston, MA 02129

The $\beta$-site amyloid precursor protein cleaving enzyme 1 (BACE1) is necessary to generate the A $\beta$ peptide, which is implicated in Alzheimer's disease pathology. Studies show that the expression of BACE1 and its protease activity are tightly regulated, but the physiological function of BACE1 remains poorly understood. Recently, numerous axon guidance proteins were identified as potential substrates of BACE1. Here, we examined the consequences of loss of BACE1 function in a well-defined in vivo model system of axon guidance, mouse olfactory sensory neurons (OSNs). The BACE1 protein resides predominantly in proximal segment and the termini of OSN axons, and the expression of BACE1 inversely correlates with odor-evoked neural activity. The precision of targeting of OSN axons is disturbed in both BACE1 null and, surprisingly, in BACE1 heterozygous mice. We propose that BACE1 cleavage of axon guidance proteins is essential to maintain the connectivity of OSNs in vivo.

T he intricate patterns of connectivity present in circuits in the mammalian nervous system provide a structural foundation for network activities that govern behavior ${ }^{1}$. Cell surface molecules are arrayed on neurons, astrocytes, and supporting cells in temporally and spatially restricted programs to achieve these connectivity patterns. By liberating domains of cell surface molecules, membrane bound proteases contribute to the complex, dynamic environment necessary to guide axons to their intended targets in the developing nervous system and maintain these circuits in the mature nervous system ${ }^{2,3}$. The BACE1 protein is a type I transmembrane aspartic protease that was discovered as the initial cleavage enzyme of the amyloid precursor protein to generate the $\mathrm{A} \beta$ peptide ${ }^{4-8}$. Subsequent studies have identified other substrates of BACE1, but its physiologic function has not been fully elucidated. The activity of the BACE1 gene product is tightly regulated by multiple levels of control: transcriptional, splicing, translational, and posttranslational modifications, as well as intracellular trafficking'. BACE1 is expressed at high levels during development and in the early postnatal period in the central nervous system, but its level of expression is significantly reduced in the adult nervous system ${ }^{10}$. However, oxidative, ischemic, and metabolic stresses lead to increased levels of BACE1 in adult neurons ${ }^{11}$. Thus, BACE1 expression corresponds with periods of structural plasticity of neural circuits. A recent proteomic study of substrates for BACE1 revealed 68 cell surface molecules as possible substrates ${ }^{12}$. Many of these proteins have a documented role in axon guidance and maintenance, e.g. semaphorins, EphA receptors, ephrins, and protocadherins. Moreover, the BACE1 cleaved ectodomain of APP, after a second cleavage, accelerates pruning of motor neuron axons and of retinal axons that target the superior colliculus during development ${ }^{13}$.

To test the hypothesis that BACE1 plays an important role in axon function that underlies the structural plasticity of neural circuits, we examined olfactory sensory neuron (OSNs) in mice that were either heterozygous or null for the BACE1 gene. The peripheral olfactory neural circuit undergoes continuous structural plasticity throughout the lifetime of the mouse ${ }^{14-16}$. In the peripheral olfactory neural circuit, (OSN) axons converge to form a spatial map of olfactory receptor-evoked activity on the surface of the olfactory bulb ${ }^{17}$. Primary olfactory neurons express just one allele of one olfactory receptor gene from a family of over 1000 olfactory genes. To a first approximation this choice is stochastic, so that patterns of activation on the surface of the olfactory epithelium by a given odorant varies greatly between individual mice. However, the olfactory receptor protein also instructs the projection of OSN axon termini to specific loci on the surface of the olfactory bulb, where they synapse with second order olfactory neurons that are dedicated to one locus. This map is functional; disruption of the projection pattern of OSNs erodes olfactory acuity in mice ${ }^{18}$.

We find that the BACE1 protein is present predominantly in the proximal axons and distal axon termini of mouse OSNs - locations of axon sorting and synaptogenesis, respectively, that are important for the establishment 
and maintenance of the map. Moreover, the precise connectivity of OSN axons from the olfactory epithelium to the olfactory bulb is disturbed in both BACE1 heterozygous and BACE1 null mice during their juvenile and adult stages of life. These results implicate an important physiological role for BACE1 activity in the function of axons in a neural circuit with documented plasticity.

\section{Results}

Expression of BACE1 in the mouse peripheral olfactory neural circuit is activity dependent. Immunostaining of mouse olfactory epithelial sections using an antibody against the BACE1 protein revealed modest expression in the dendrites and soma of OSNs (Fig. 1a). However, robust staining was seen in the axon bundles of OSNs which costain with NCAM (Fig. 1c). In the mouse olfactory bulb, the antibody recognizing BACE1 labelled the glomeruli in the olfactory bulb (Fig. 1d), which is consistent with previous findings in the rat olfactory bulb ${ }^{19}$. Immunostaining of heterozygous or null BACE1 mice ${ }^{20}$ revealed labeling reduced about $50 \%$ (Fig. 1e) and $100 \%$ (Fig. 1f), respectively (Figure 1g). We examined BACE1 expression in primary olfactory neurons in the olfactory epithelium of mice using in situ hybridization. The level of expression was present throughout the lamina, suggesting that both immature and mature OSNs express BACE1 mRNA, with an slight increase in mature OSNs (Fig. 1h). In addition, we observed a smooth gradient of expression with the greatest levels of expression in the ventrolateral regions and the least expression in the dorsomedial regions. In the olfactory bulb, we detected BACE1 mRNA in periglomerular interneurons and second order olfactory neurons (mitral cells). No signal was detected by probing with the sense strand (data not shown).

Previous work in the rat olfactory bulb indicated an inverse relationship between the level of BACE1 protein and odor-evoked activity ${ }^{19}$. Since BACE1 is regulated by transcriptional and translational mechanisms, we determined whether naris closure alters BACE1 mRNA and proteins levels in wild type mice. Mice, who underwent naris closure at $\mathrm{P} 0$, were analyzed at 2 weeks of age. BACE1 mRNA was substantially increased in the dorsomedial region of the olfactory epithelium (Fig. 1j) and olfactory bulb (Fig. 1k) on the occluded side, thereby abrogating the gradient of expression. Moreover, BACE1 protein was increased in the glomeruli on the occluded side, particularly in the dorsomedial glomeruli (Fig. 11), paralleling the pattern of changes of BACE1 mRNA expression in the olfactory epithelium. Quantification of BACE1 protein expression in the glomeruli revealed statistically significant differences in the dorsal, medial, and lateral regions of the bulb (Fig. $1 \mathrm{~m}$ ). These olfactory bulb data are consistent with the findings of increased BACE1 protein following naris closure in the glomeruli of 7 to 8 month old mice that overexpress a pathogenic APP allele ${ }^{21}$.

BACE1 protein is present in the proximal axons and axon termini of OSNs. To localize the BACE1 protein in the glomeruli in the olfactory bulb with subcellular resolution, we conducted multiple costaining experiments with the specific markers of different cell types that contribute to the neuropil within glomeruli. Specifically, we costained with markers for axons (NCAM; Fig. 2a - d), OSNs (OMP; Fig. 2e - h), interneurons (GAD65; Fig. 2i - 1), dendrites of second-order neurons (MAP2; Fig. $2 \mathrm{~m}-$ p), postsynaptic densities (PSD95; Fig. $2 \mathrm{q}-\mathrm{t}$ ) in the glomeruli. Each marker was compared with the relevant non-glomerular layer (ONL for axonal markers and EPL for dendritic markers). The olfactory marker protein (OMP) of olfactory sensory axons exhibited a significant degree of overlap with the BACE1 labeling pattern (Fig. 2h), as did the axonal marker NCAM (Fig. 2d). By contrast, the dendrites of second order neurons (MAP2; Fig $2 \mathrm{~m}-\mathrm{o}$ ) exhibited an insignificant degree of overlap with the BACE1 labeling pattern (Figure 2p). Overlap with the interneuron marker (GAD65; Fig. 2l) was also significant in the glomerulus relative to the OPL. BACE1 immunostaining significantly overlapped with staining of the postsynaptic marker (PSD95: Fig. 2t). Taken together, these data indicate that BACE1 is expressed in the proximal (Fig. 1a) axon bundles and distal (Fig. 2) axon termini of presynaptic OSNs near sites of synapses. Without EM characterization, we cannot quantitatively determine the relative proportions of BACE1 expression in primary, interneuron, and second-order neuron post-synaptic compartments within a glomerulus, but our qualitative data suggests that BACE1 expression is enriched in axonal compartments.

Axon mistargeting of OSNs in BACE1 heterozygous and BACE1 null mice in a dorsal to ventral gradient. We examined the precision of OSN axon targeting in littermate BACE1 null, BACE1 heterozygous and wild type mice. The age ranges were from 1 to 3 months. To visualize OSN axon projection patterns, we immunostained the olfactory bulbs with antibodies against the OR M71. The convergence of OSN axons expressing the M71 OR was disturbed in both BACE1 heterozygous and BACE1 null mice (Figure $3 \mathrm{a}-\mathrm{d} ; \mathrm{p}<0.01$ ). To determine whether BACE1 deficiency influenced other subpopulations of OSNs, we crossed the BACE1 null mice with mice coexpressing GFP from one allele of the P2 olfactory receptor ${ }^{22}$. Analysis of littermate wild type, BACE1 haploinsufficient, and BACE1 null mice showed a greater degree of axon dispersion of $\mathrm{P} 2$-expressing OSNs in the BACE1 null mice relative to the BACE1 heterozygous and wild type littermates (Fig. $3 \mathrm{e}-\mathrm{h} ; \mathrm{p}<0.01$ ). By contrast, analysis of the BACE1deficient mice crossed with a mouse line expressing a marker protein coexpressed with the MOR28 olfactory receptor showed no alteration in OSN axon targeting (Fig. $3 \mathrm{i}-3 \mathrm{l}$ ). These three olfactory receptors project to different regions of the olfactory bulb, suggesting a regional importance for the role of BACE1 in the precision of axon targeting. In the dorsal olfactory bulb, M71-expressing OSNs exhibit a significant difference between wild type and heterozygous BACE1 mice as well as heterozygous BACE1 and BACE1 null mice. For $\mathrm{P} 2$-expressing OSNs in the equatorial bulb, BACE1 null mice are significantly different from both BACE1 heterzygous and wild type mice, but BACE1 heterozygous mice are not significantly different from BACE1 null mice. The axons of ventral MOR28-expressing OSNs targeted correctly.

Alterations in substrates in BACE1 deficient mice. APP and the amyloid precursor like protein 2 (APLP2) are validated substrates for BACE1 and are expressed by OSNs ${ }^{9}$. APP is significantly increased (Fig. 4d) in the glomeruli of the olfactory bulbs of both BACE1 heterozygous and BACE1 null mice as revealed by immunostaining with an antibody that recognizes the COOH-terminus (Fig. $4 \mathrm{a}-\mathrm{c}$ ). A regional analysis of the olfactory bulb indicates a significant increase in all quadrants of the BACE1 null mice. Interestingly, the ventral and lateral regions are not significantly (Fig. 4e) increased in the BACE1 heterozygous mice, which receive OSN axons from ventral and lateral regions of the olfactory epithelium. The ambient levels of BACE1 expression is greater in these regions, and the levels are not modulated by odor evoked activity (Fig. 1j). Additionally, APLP2 levels are significantly increased (Fig. 4i) in the outer nerve layer and glomeruli of the olfactory bulbs in both BACE1 heterozygous and BACE1 null mice (Fig. 4f-h) as revealed by an antibody that recognizes the full length of APLP2. Similarly, all four quadrants show a significant increase between BACE1 null and littermate controls; however, only the ventral region does not show statistical significance between the BACE1 heterozygous mice and littermate wild type controls (Fig. 4j). Together, these data indicate that the BACE1 heterozygous mice are haploinsufficient, an intriguing finding for an enzyme without known imprinting.

Reduced odor-evoked gene induction in BACE1 deficient mice. To determine whether the OSN axon mistargeting in BACE1 

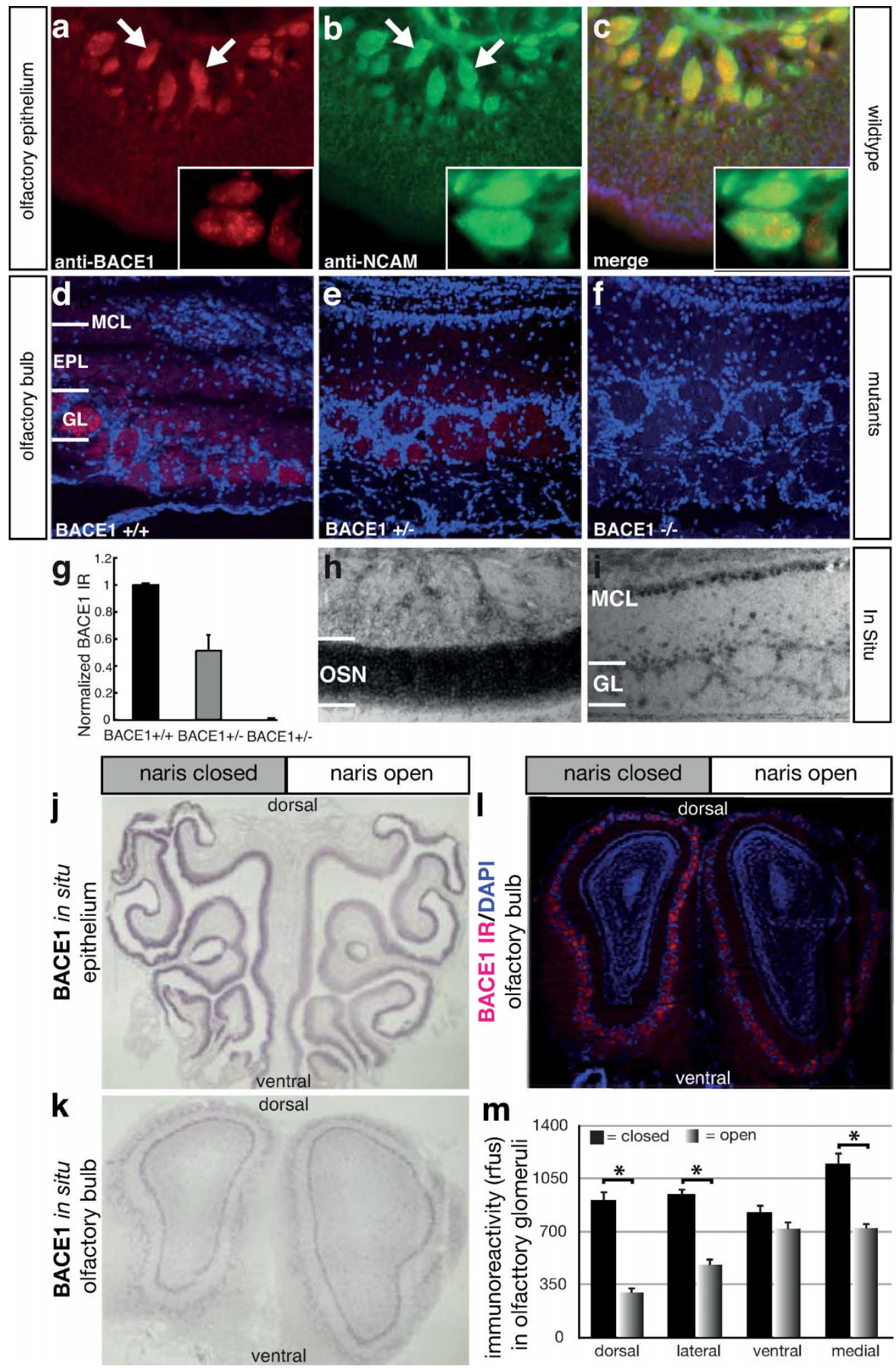

m

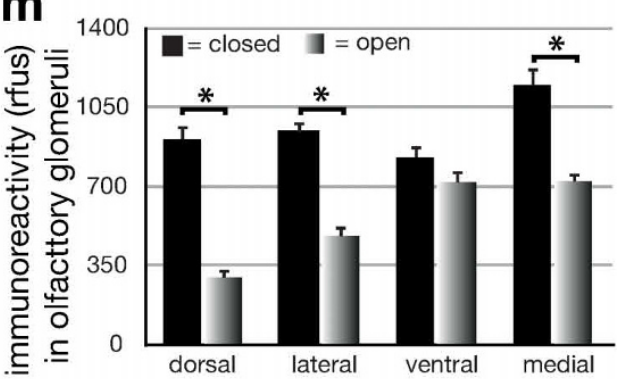

Figure 1 BACE1 expression in mouse olfactory epithelium and olfactory bulb is activity dependent. (a-c) BACE1 immunoreactivity (IR) is enriched in the axon bundles (arrows) of olfactory sensory neurons (OSNs), where it is colocalized with NCAM, an axon-specific marker protein. The insets indicate that BACE1 is partially colocalized with NCAM in the axon bundles. (d) BACE1 IR is present in the olfactory bulb, with the highest level in the glomerular layer (GL), a modest level in the external plexiform layer (EPL) and absent in the mitral cell layer (MCL). (e-f) BACE1 IR is reduced in BACE1 heterozygous mice (BACE1 $+/-$ ) and absent in BACE1 homozygous knockout mice (BACE1 -/-). (g) Quantification of BACE1 IR intensity in the olfactory bulb. ( $\mathrm{h}-\mathrm{i}$ ) In situ hybridization reveals graded levels of BACE1 mRNA in the olfactory epithelium (h) and robust staining in the mitral cell layer of the olfactory bulb (i). Unilateral naris closure in control mice reveals a marked increase in the BACE1 mRNA levels on the closed side of the olfactory epithelium (j) but not in the mitral cells of the olfactory bulb (k). Quantitation of the BACE1 protein levels in the glomeruli of the olfactory bulb (l) reveals significant increases $(\mathrm{m})$ in the dorsal, medial, and lateral regions of the olfactory bulb $(\mathrm{p}<0.001)$. Black bars represent closed side, and gradient bars represent the open side. 


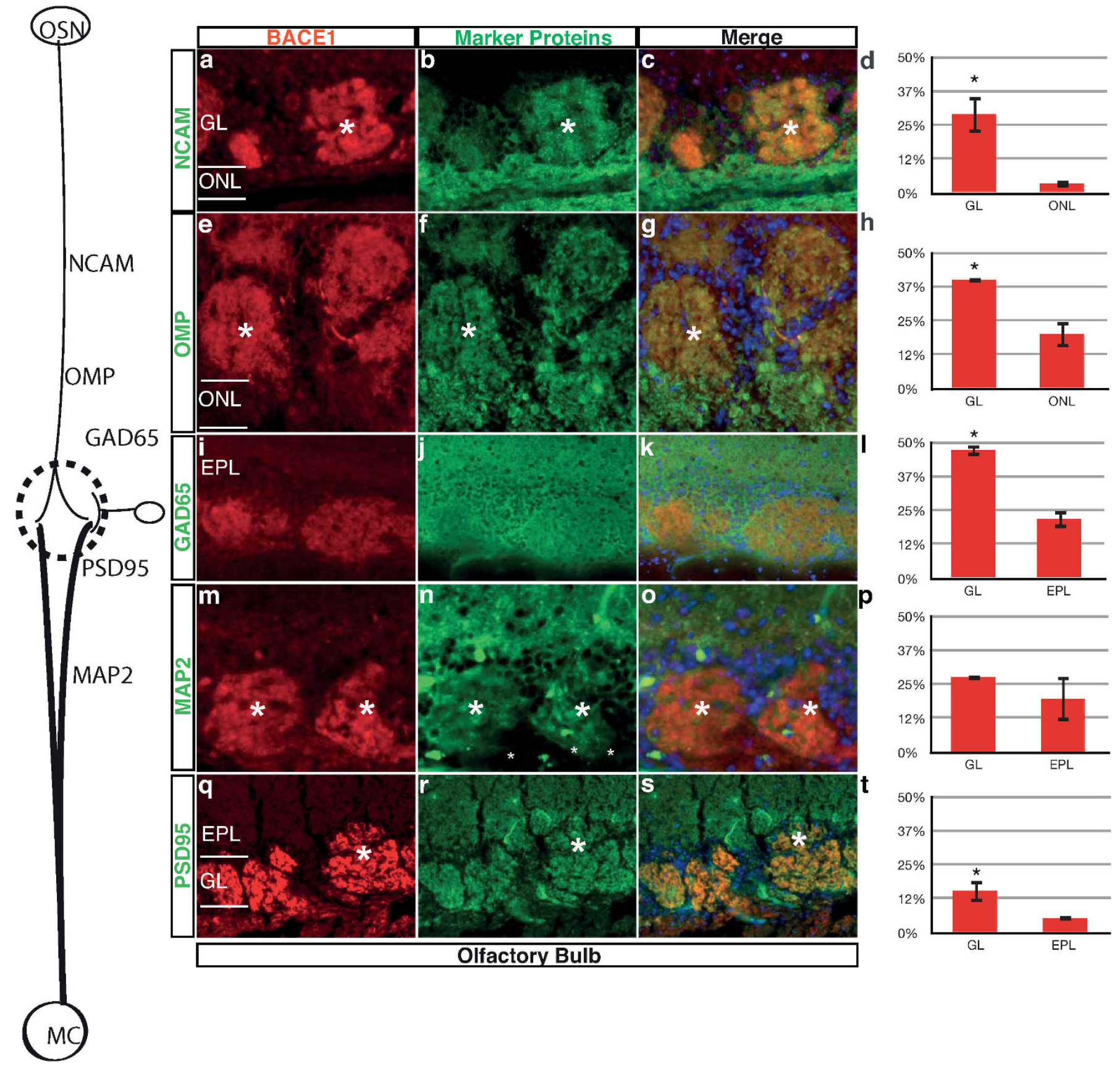

Figure 2 BACE1 IR predominantly colocalizes with pre-synaptic neuronal marker proteins in the mouse olfactory bulb. (a - c) Colocalization of BACE1 IR and NCAM IR in glomerular layer (GL) but not in the outer nerver layer (ONL). (d) Percentage of overlapping pixels of NCAM and BACE1 in GL and ONL. (e-g) BACE1 IR overlaps with OMP IR, a specific marker of OSNs, in GL. (h) Percentage of overlapping pixels of OMP and BACE1. (i-k) BACE1 IR overlaps with GAD65 IR, a specific marker of inhibitory periglomerular cells in GL. (1) Percentage of overlapping pixels of of GAD65 and BACE1 revealed that it is higher in GL than that in external plexiform layer (EPL). ( $\mathrm{m}-\mathrm{o}$ ) Colocalization of BACE1 IR with MAP2 (a dendritic marker protein) immunoreactivity in GL and OPL. (p) Quantification of the overlapping level of MAP2 and BACE1. (q-s) BACE1 colocalizes with postsynaptic protein PSD95 in GL and EPL. (t) Quantification of the overlapping level of PSD95 and BACE1. ${ }^{*}=\mathrm{p}<0.05$.

deficient mice has functional implications, we immunostained periglomerular neurons for tyrosine hydroxylase $(\mathrm{TH})$. The levels of expression of this gene is proportional to input from $\mathrm{OSNs}^{23,24}$. Immunostaining of the olfactory bulbs demonstrate reduced induction of TH in BACE1 null but not BACE1 haploinsufficient mice (Fig. 5), although a trend was present for the BACE1 haploinsufficient mice. Consistent with the dorsal to ventral gradient of axon mistargeting, we observe a dorsal to ventral loss of $\mathrm{TH}$ induction in the olfactory bulb. This concordance supports the notion that alterations in the fidelity of OSN axon targeting alter perceived activity postsynaptically in BACE1-deficient mice. To exclude the possibility that a loss of OSNs underlies the reduced TH induction in BACE1 null mice, we quantified subpopulations of OSNs defined by the olfactory receptors M71, P2, and MOR28. No significant difference was observed in BACE1 null or BACE1 haploinsufficient mice relative to littermate controls (Fig. 6a-c). Additionally, we did not find evidence of increased rate of neurogenesis in the olfactory epithelium as evidenced by expression of BrdU labeling (Fig. 6d) or increased density of cleaved caspase 3 immunostaining (data not shown). Together these experiments indicate that the connectivity disturbance is not a consequence of accelerated turnover of OSNs in the olfactory epithelium. 
BACE1 +/+

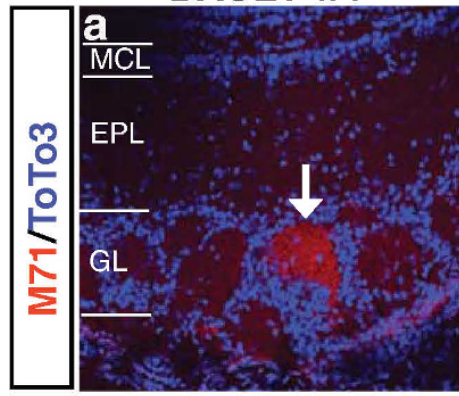

이을

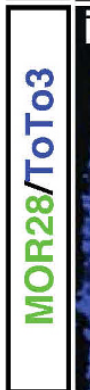

BACE1 +/-
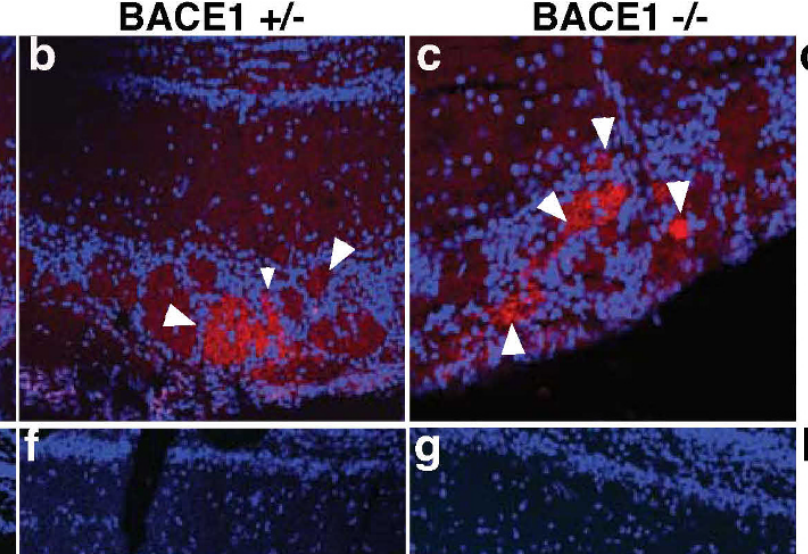

$\mathbf{d}_{\text {을 }}$
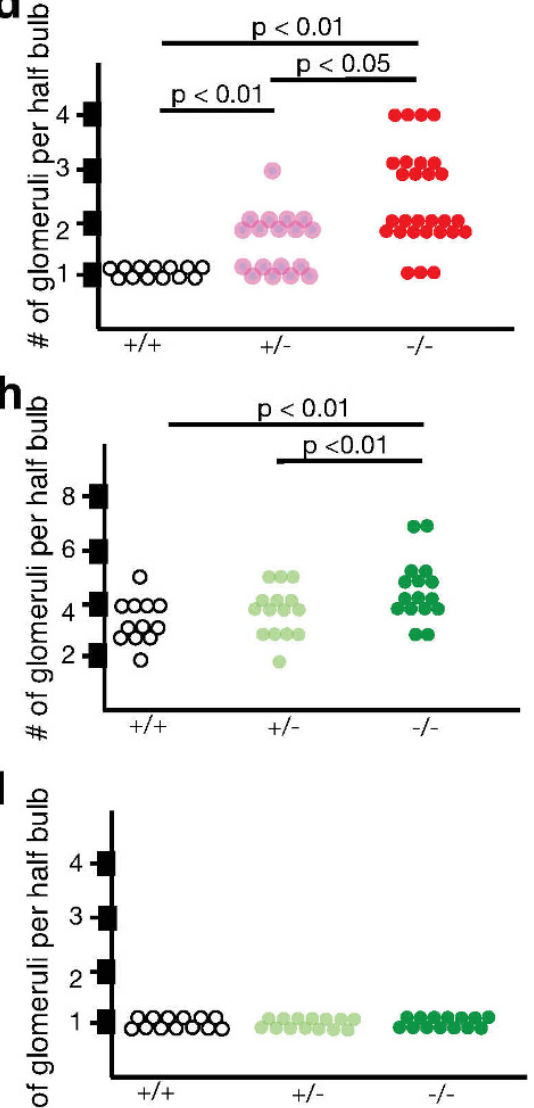

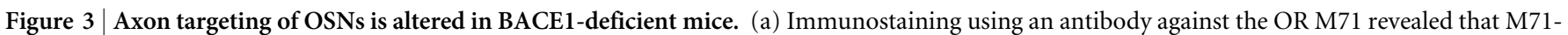

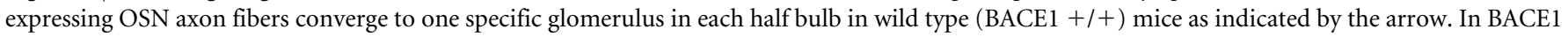

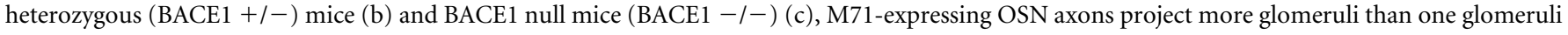

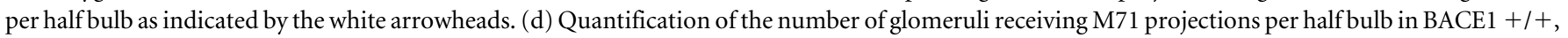

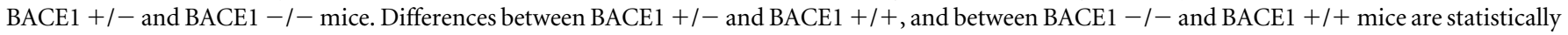

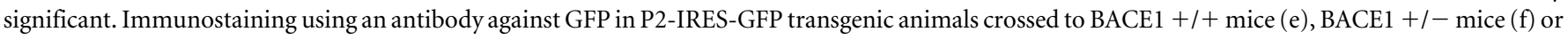

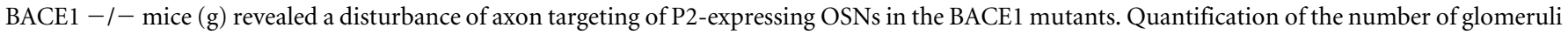

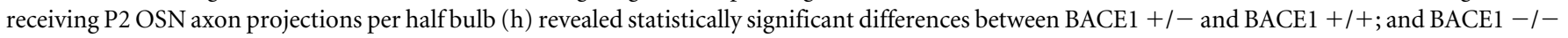

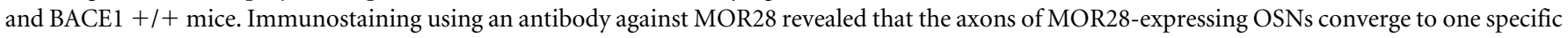
glomerulus in each half bulb of the BACE1 mutants (i - k). Quantification of the number of glomeruli receiving MOR28-expressing OSN axon

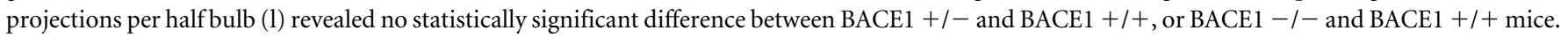

\section{Discussion}

The emerging evidence supporting a role for membrane associated proteases in axon guidance have focused on metalloproteases and $\gamma$ secretases in both invertebrate and vertebrate organisms ${ }^{2,3}$. To test the hypothesis that the membrane-associated protease BACE1 also plays a role in guiding axons in vivo, we analyzed the connectivity of OSNs in mice with only one or no BACE1 functional allele. The expression of the BACE1 gene in the mouse olfactory epithelium is graded with less BACE1 mRNA present in the dorsal and medial regions. This dorsomedial area does not conform to the strict boundaries of zone $1^{25,26}$. It is suggestive of regions of hydrophilic odor absorption in the rat nose and may be related to neuronal activity ${ }^{27}$. Naris closure abrogates this gradient by increasing expression in the medial and dorsal regions, providing additional evidence that odorevoked activity suppresses BACE1 gene expression ${ }^{19}$.

Mouse OSNs are arranged in vivo with the soma and proximal axons virtually bisected from the distal axons by the cribiform plate. Employing this landmark we find that the BACE1 gene product resides in the proximal segment of OSN axons and in distal axon termini in the glomeruli. Moreover, axons of OSNs form an orderly, reproducible projection pattern ${ }^{17}$. This mapping of axons primarily depends on the identity of the neurons, as defined by their choice of olfactory receptor, and secondarily upon the spatial relationship of their soma. We find that OSNs expressing the same olfactory receptor do not project to the same glomerulus, which is consistent with role for the BACE1 protease in axon targeting. We cannot distinguish between the scenarios that the axons do interpret the cues en route to their target with more errors or a deficit of pruning mistargeted fibers. Indeed, a recent study that a BACE1-dependent cleavage product of APP is involved in axon pruning in spinal cord neurons and retinotectal projections ${ }^{13}$. If this mechanism were operative, we might expect evidence of diminished turnover of OSNs in the epithelium of BACE1 deficient mice. However, the rates of neurogenesis, apoptosis, and steady state levels of olfactory receptor-defined subpopulations are unchanged in BACE1 haploinsufficient or null mice.

We favor the hypothesis that OSN axons are misinterpreting the cues, perhaps by alterations of the levels of cell surface molecules and/or modulating the production of physiological active cleavage products. In BACE1 deficient mice we observed an increase (or 

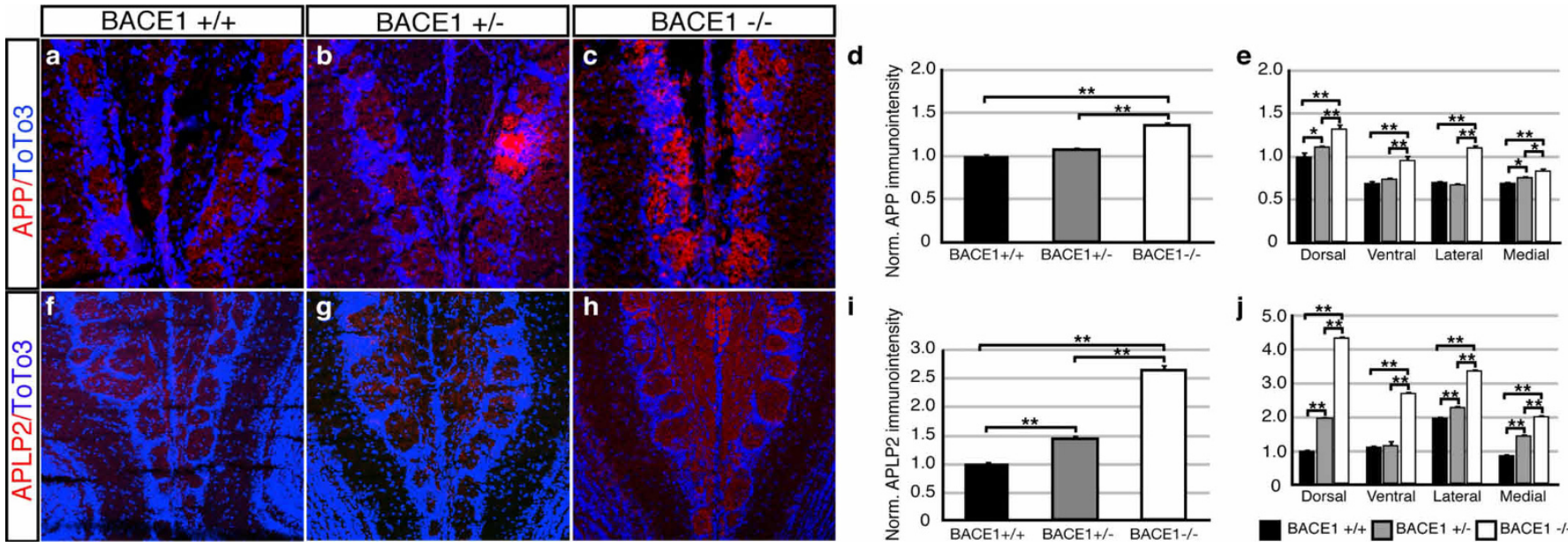

Figure $4 \mid$ Increased presence of APP and APLP2 in olfactory bulbs of BACE1 heterozygous and null mice. Representative images of immunostaining for APP in olfactory bulbs from littermate wild type (a), BACE1 +/- (b), and BACE1 - / - (c). Quantification of the intensity of immunostaining (d) revealed a significant difference between the BACE1 - / - with either the wild type or BACE $1+/-$ mice ( $\mathrm{n}=3$ sets of 3 littermates). A regional analysis of immunostaining (e) in the glomeruli of the olfactory bulb reveals significant increases in APP staining in the ventral and lateral regions. Representative images of immunostaining for APLP2 in olfactory bulbs from littermate wild type (f), BACE1 $+/-(\mathrm{g})$, and BACE1 - $-(\mathrm{h})$. Quantification of the intensity of immunostaining (i) revealed a significant difference between the BACE1 $-/-$ with either the wild type or BACE $1+/-$ mice ( $\mathrm{n}=3$ sets of 3 littermates). A regional analysis of immunostaining $(\mathrm{j})$ in the glomeruli of the olfactory bulb reveals significant increases in APLP2 staining in all 4 regions. $*=\mathrm{p}<0.05 ; * *=\mathrm{p}<0.01$.

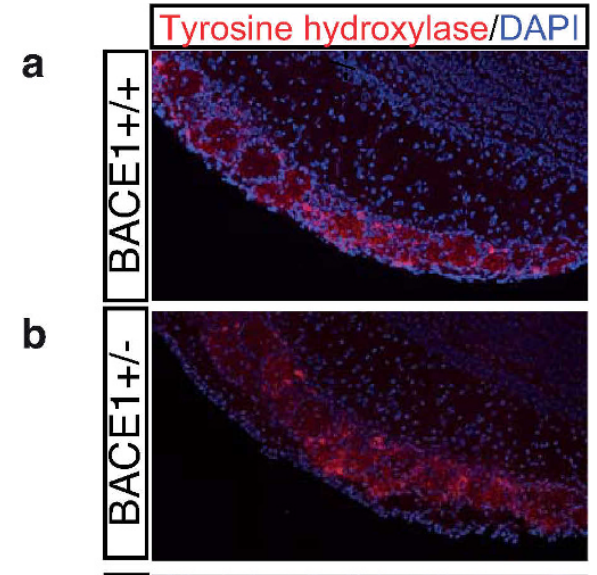

C

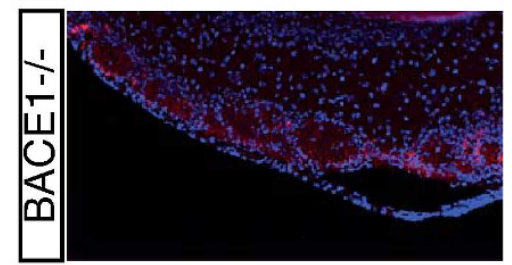

d

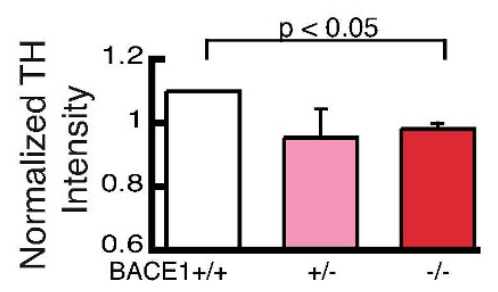

Figure $5 \mid$ Reduced tyrosine hydroxylase induction in BACE1 null mice. Tyrosine hydroxylase (TH) levels correlate with OSN input into glomeruli. Representative images of olfactory bulbs immunostained with an antibody recognizing TH (red) and a nuclear stain DAPI (blue) from a control BACE1 $+/+$ mouse (a), a BACE1 + / - mouse (b), and a BACE1 - /mouse (c). Quantitation of the intensity of TH staining in glomeruli of BACE1 deficient mice $(\mathrm{n}=4)$ and wild type littermate mice $(\mathrm{n}=4)$ reveals a statistically significant difference $(\mathrm{p}<0.05)$ between BACE1 $+/+$ and BACE1 $-/-$ mice $(\mathrm{d})$. redistribution) of APP and APLP2 in the axons and axon termini of OSNs. Modulation of APP and APLP2 have been implicated in neurite outgrowth ${ }^{28}$, cortical neuron migration ${ }^{29}$, and motor neuron axon targeting $^{30}$. We have evidence that loss of function of APP causes a disruption of the connectivity of the mouse peripheral olfactory neural circuit (L.C, G.T.R., S.R., T.W.M., M.W.A., in preparation). Several proposed substrates for BACE1, e.g., ephrin-A5 and protocadherins, are cell surface molecules with defined roles in axon guidance of mouse olfactory sensory neurons ${ }^{31,32}$. We also observe increased levels of ephrin expression, as evidenced by EphA4 ectodomain binding revealed by alkaline phosphatase stain (data not shown $)^{32}$. Elegant genetic studies have demonstrated the
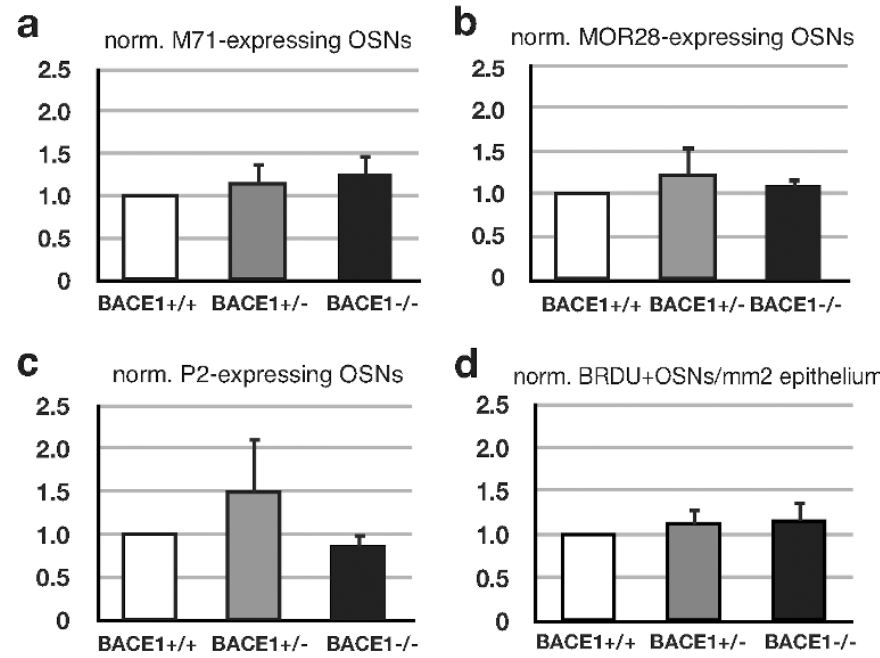

Figure 6 | No significant loss of specific OSN populations or altered rates of neurogenesis in BACE1 null and heterozygous mice. Normalized counts of genetically defined subpopulations of M71- (a), MOR28- (b), or P2- (c) expressing OSNs in a systematic series of sections spanning the entire olfactory epithelium are not significantly different $(\mathrm{p}>0.05$, mutants vs. control, $\mathrm{n}=4$ ). (d) Quantitation of the normalized density of BrdU-positive OSNs in a series of sections spanning the entire olfactory epithelium in BACE1 $-/-$, BACE1 $+/-$ and wild type control mice reveals no significant difference ( $\mathrm{p}>0.05$, mutants vs. control, $\mathrm{n}=4)$. 
role of the neuropilins and their semaphorin ligands (family members are candidate BACE1 substrates) $)^{12}$, in the establishment of the map of OSN axon projections $s^{33,34,35}$. Specifically, neuropilin-1 and its ligand semaphorin $3 \mathrm{~A}$ mediate axon sorting in the proximal OSN axon bundles ${ }^{36}$, a site of high BACE1 expression.

Both genetic and biochemical evidence implicate the generation of the $A ß$ peptide as playing a central role in the pathogenesis of earlyonset Alzheimer's disease $(\mathrm{AD})^{37,38}$. Mice with deletions of the $B A C E 1$ gene do not produce $A ß$ when they are crossed with mice overexpressing human APP in the brain ${ }^{39}$. The amyloid pathology, memory deficits, cholinergic dysfunction in the hippocampus, and neuronal loss seen in the $5 \mathrm{x}$-FAD line, which overexpress human APP and presenilin 1 proteins with several pathogenic mutations, were absent in $5 \times$ FAD mice on a BACE1 null background ${ }^{40}$. Thus, $B A C E 1$ is essential for $A ß$ production in mice, and inhibitors of BACE1 are under development as potential therapeutic targets in humans.

One implication of this study is that molecules that inhibit BACE1 function may have the undesired side effect of altering neural connections in brain regions with structural plasticity. BACE1 null mice exhibit alterations in long term potentiation as well as memory and emotional behavioral tasks, all of which depend on structural plasticity of neural circuits ${ }^{20}$. One uncommon feature of the olfactory neural circuit is ongoing neurogenesis throughout the life of the organism. However, continuous neurogenesis is also a feature of the dentate gyrus of the hippocampus. It is difficult to assess the precision of connectivity in the dentate gyrus since cell-type specific markers that define the projection target are not yet available. Nevertheless, it remains possible that axon mistargeting in the hippocampal neural circuit accounts in part for the learning and electrophysiological deficits documented in BACE1 null animals ${ }^{20}$. In addition, BACE1 is also upregulated after neuronal injury from ischemic or traumatic insults ${ }^{41}$. Inhibition of BACE1 function may hamper the repair or recovery process by altering the plasticity responses to these insults. Our results demonstrating connectivity phenotypes in a neural circuit with defined plasticity in the BACE1 haploinsufficient mouse raise an element of caution about the therapeutic strategy to partially inhibit BACE1, and provide impetus for the development of substrate-specific BACE1 modulators. Future studies employing directed recombination to abrogate BACE1 expression in restricted populations of neurons will elucidate its function with finer resolution in the context of neural circuits in vivo.

In summary, genetic reduction or elimination of the BACE1 protein results in alterations in structural connectivity of the mouse peripheral olfactory neural circuit. Recent studies revealed that order emerges in the axon bundles of OSNs by precise sorting to form fascicles that generate the spatial projection map in the olfactory bulb $^{33}$. Our data are consistent with a model that BACE1 regulates the spatial and temporal code of cell surface molecules that afford identity-driven sorting of axon fascicles and their targeting. Future studies will be directed at identifying the BACE1 substrates that mediate OSN axon sorting and the precise role of BACE1 cleavage of these substrates, e.g. downregulation of cell surface molecules and/ or unleashing biologically active fragments. More generally, recent studies have suggested additional roles for axon guidance molecules in the mature nervous system, such as axon pruning and cell death ${ }^{42}$. Further studies will be focused on defining the role that BACE1 function plays, if any, in orchestrating these processes.

\section{Methods}

Animals. All experiments were in accordance with protocols approved by the Institutional Animal Care and Use Committee of Massachusetts General Hospital. BACE1 null ${ }^{43}$ were obtained from Jackson Labs (Bar Harbor, ME). P2-ires-GFP 22 mice were a gift from Richard Axel (Columbia University). Naris closure was performed on P0 mice as previously described ${ }^{44}$. To generate tissue blocks for sectioning, the mice were anesthetized with an intraperitoneal injection of 3,3, 3-tribromoethanol (1.25\% in PBS $30 \mu \mathrm{L} / \mathrm{g}$ body weight). Following thoracotomy, intracardiac perfusion with $10 \mathrm{~mL}$ of $\mathrm{PBS}$ ( $\mathrm{pH} 7.4$ ) was performed.
In situ hybridization. Twenty $\mu \mathrm{m}$ thick fresh frozen tissue sections were placed on Superfrost slides (Fisher Scientific). The sections were dried for $45 \mathrm{~min}$. at room temperature before fixing with $4 \%$ paraformaldehyde for $15 \mathrm{~min}$., and washed three times in $1 \mathrm{x}$ DEPC-treated PBS containing $1 \mathrm{mM} \mathrm{MgCl}_{2}$. Slides were then immersed in a solution containing $270 \mathrm{~mL}$ of DEPC treated water, $30 \mathrm{~mL}$ of $1 \mathrm{M}$ triethanolamine and $750 \mu \mathrm{L}$ of $95 \%$ acetic anhydride for 10 minutes, and subsequently washed $3 \mathrm{x}$ in $1 \mathrm{x}$ DEPC-treated PBS containing $1 \mathrm{mM} \mathrm{MgCl}$. Slides were then blocked for 2 hours with hyridization buffer: $0.1 \%$ Tween $20,50 \%$ formamide, 5x SSC, $5 x$ Denhardts, 5 mM EDTA, $10 \mathrm{mM} \mathrm{NaH} 2 \mathrm{PO} 4$ at $\mathrm{pH}$. 8.0, $50 \mathrm{mM}$ Tris $\mathrm{pH}$ 8.0, $250 \mu \mathrm{g} / \mathrm{mL}$ salmon sperm DNA, $100 \mu \mathrm{gram} / \mathrm{mL}$ tRNA, $100 \mu \mathrm{g} / \mathrm{mL}$ yeast RNA. Slides were dabbed dry and RNA probes $(100 \mu \mathrm{g} / \mathrm{mL})$, preheated for $5 \mathrm{~min}$. at $80^{\circ} \mathrm{C}$ and cooled on ice for $2 \mathrm{~min}$., and were applied to the slides. The slides were sealed in a humidified chamber and incubated at $65^{\circ} \mathrm{C}$ overnight. After $18 \mathrm{~h}$ the slides were washed three times in $5 \mathrm{xSSC}$ at $65^{\circ} \mathrm{C}$ for $15 \mathrm{~min}$., and then three times with $0.2 \mathrm{x}$ SSC for $20 \mathrm{~min}$. After blocking in $1 \mathrm{x}$ in situ hybridization blocking solution (Roche) for $1 \mathrm{~h}$, the slides were dabbed dry and a sheep antibody recognizing digoxetin $(1: 3000$, Roche) was applied overnight. The next day the RNA probe was detected using the HNPP fluorescent detection kit (Roche) or BCIP/NBT (Promega).

Immunohistochemistry. For immunostaining using antibodies against the olfactory receptors (ORs) MOR28 and M71, the sections were post-fixed with $1 \%$ paraformaldehyde in PBS for $8 \mathrm{~min}$. at room temperature. For the other antibodies, the mice were subsequently perfused with $4 \%$ paraformaldehyde in PBS (pH 7.4). The olfactory turbinates and olfactory bulbs were dissected intact, incubated in $30 \%$ sucrose in PBS at $4^{\circ} \mathrm{C}$ overnight, and embedded in OCT (Sakura) or M1 (Shandon) in a dry ice/ethanol bath. Twenty $\mu \mathrm{m}$ coronal sections were cut on a cryostat (Microm) and collected on SuperFrost slides (Fisher). All sections were washed three times in PBS for 10 min., permeabilized in $0.1 \%$ Triton X-100 in PBS (PT) for $30 \mathrm{~min}$. at room temperature, blocked in 5\% heat-inactivated horse serum in PT (PTS) for $1 \mathrm{~h}$ at room temperature, incubated with primary antibody in PTS under a Hybrislip (Invitrogen) overnight in a humidified chamber at $4^{\circ} \mathrm{C}$, washed three times with PT for $10 \mathrm{~min}$., blocked with PTS for $30 \mathrm{~min}$. at room temperature, incubated with a fluorescentconjugated secondary antibody in PTS with TOTO-3 (Invitrogen, 1:1000) or DAPI (Invitrogen $100 \mathrm{ng} / \mathrm{mL}$ ) for $2 \mathrm{~h}$ at room temperature, washed briefly in PBS, and Vectashield was applied to each slide and coverslipped. Slides were imaged using a Zeiss LSM-510 confocal microscope, or a Zeiss inverted fluorescent microscope and analyzed using Image $(\mathrm{NIH})$.

Primary antibodies included rabbit anti-BACE1 (Cell Signaling, $1: 500$ ), rabbit anti-GFP (Molecular Probes; $1: 1000$ ), sheep anti-GFP (Biogenesis; $1: 1000)$, rabbit anti-LacZ (Cappel; $1: 1000)$, rabbit anti-MOR28 (1:3000) (Barnea et al., 2004), guinea pig anti-M71 ( $1: 1000)$ (Barnea et al., 2004), rabbit anti-APLP2 (Calbiochem; $1: 500$ ), rabbit anti-APP (Zymed; $1: 300$ ), rabbit anti-activated caspase 3 (Cell Signaling; $1: 500$ ), mouse anti-BrdU (Invitrogen; $1: 1000$ ), mouse anti-reelin (Developmental Studies Hybridoma Bank of University of Iowa; $1: 10$ ), mouse antityrosine hydroxylase (Millipore; $1: 500$ ), mouse anti-PSD95 (Neuromap; $1: 200$ ), mouse anti-NCAM (Sigma; $1: 300)$, goat anti-OMP (Wako; $1: 500)$, mouse antiGAD65 (BD; $1: 100$ ), and mouse anti-MAP2 (Sigma, 1:100). Secondary antibodies were Alexa 488-conjugated or Cy3-conjugated donkey anti-rabbit IgG (Molecular Probes; $1: 500$ ), Alexa488-conjugated donkey anti-sheep IgG (Molecular Probes; $1: 500$ ), Cy3-conjugated donkey anti-guinea pig IgG (Jackson Immunoresearch; $1: 500$ ), Alexa 488-conjugated or Cy3-conjugated donkey anti-mouse IgG (Jackson Immunoresearch; $1: 500$ ) and Cy3-conjugated donkey anti-rabbit IgG (Jackson Immunoresearch; $1: 500$ ).

Statistical Analyses. One-way analysis of variance and unpaired t-tests were used, as appropriate. Each statistical test was performed as described in the methods and figures legends using Excel (Microsoft), or Numbers (Apple).

1. Surmeli, G., Akay, T., Ippolito, G. C., Tucker, P. W. \& Jessell, T. M. Patterns of spinal sensory-motor connectivity prescribed by a dorsoventral positional template. Cell 147, 653-665 (2011).

2. Bai, G. \& Pfaff, S. L. Protease regulation: the Yin and Yang of neural development and disease. Neuron 72, 9-21 (2011).

3. O’Donnell, M., Chance, R. K. \& Bashaw, G. J. Axon growth and guidance: receptor regulation and signal transduction. Annu Rev Neurosci 32, 383-412 (2009).

4. Vassar, R. et al. Beta-secretase cleavage of Alzheimer's amyloid precursor protein by the transmembrane aspartic protease BACE. Science 286, 735-741 (1999).

5. Hussain, I. et al. Identification of a novel aspartic protease (Asp 2) as betasecretase. Mol Cell Neurosci 14, 419-427 (1999).

6. Sinha, S. et al. Purification and cloning of amyloid precursor protein betasecretase from human brain. Nature 402, 537-540 (1999).

7. Yan, R. et al. Membrane-anchored aspartyl protease with Alzheimer's disease beta-secretase activity. Nature 402, 533-537 (1999).

8. Lin, X. et al. Human aspartic protease memapsin 2 cleaves the beta-secretase site of beta-amyloid precursor protein. Proc Natl Acad Sci U S A 97, 1456-1460 (2000).

9. Cole, S. L. \& Vassar, R. The Basic Biology of BACE1: A Key Therapeutic Target for Alzheimer's Disease. Curr Genomics 8, 509-530 (2007).

10. Hebert, S. S. et al. Loss of microRNA cluster miR-29a/b-1 in sporadic Alzheimer's disease correlates with increased BACE1/beta-secretase expression. Proc Natl Acad Sci U S A 105, 6415-6420 (2008). 
11. O'Connor, T. et al. Phosphorylation of the translation initiation factor eIF2alpha increases BACE1 levels and promotes amyloidogenesis. Neuron 60, 988-1009 (2008)

12. Hemming, M. L., Elias, J. E., Gygi, S. P. \& Selkoe, D. J. Identification of betasecretase (BACE1) substrates using quantitative proteomics. PLoS One 4, e8477 (2009).

13. Nikolaev, A., McLaughlin, T., O'Leary, D. D. \& Tessier-Lavigne, M. APP binds DR6 to trigger axon pruning and neuron death via distinct caspases. Nature 457 981-989 (2009).

14. Albers, M. W., Tabert, M. H. \& Devanand, D. P. Olfactory dysfunction as a predictor of neurodegenerative disease. Curr Neurol Neurosci Rep 6, 379-386 (2006)

15. Arnold, S. E. et al. Olfactory epithelium amyloid-beta and paired helical filamenttau pathology in Alzheimer disease. Ann Neurol 67, 462-469 (2010).

16. Talamo, B. R. et al. Pathological changes in olfactory neurons in patients with Alzheimer's disease. Nature 337, 736-739 (1989).

17. Axel, R. Scents and sensibility: a molecular logic of olfactory perception (Nobe lecture). Angew Chem Int Ed Engl 44, 6110-6127 (2005).

18. Fleischmann, A. et al. Mice with a "monoclonal nose": perturbations in an olfactory map impair odor discrimination. Neuron 60, 1068-1081 (2008).

19. Yan, X. X., Xiong, K., Luo, X. G., Struble, R. G. \& Clough, R. W. beta-Secretase expression in normal and functionally deprived rat olfactory bulbs: inverse correlation with oxidative metabolic activity. J Comp Neurol 501, 52-69 (2007).

20. Laird, F. M. et al. BACE1, a major determinant of selective vulnerability of the brain to amyloid-beta amyloidogenesis, is essential for cognitive, emotional, and synaptic functions. J Neurosci 25, 11693-11709 (2005).

21. Zhang, X. M. et al. Functional deprivation promotes amyloid plaque pathogenesis in Tg2576 mouse olfactory bulb and piriform cortex. Eur J Neurosci 31, 710-721 (2010)

22. Wang, F., Nemes, A., Mendelsohn, M. \& Axel, R. Odorant receptors govern the formation of a precise topographic map. Cell 93, 47-60 (1998).

23. Baker, H. Unilateral, neonatal olfactory deprivation alters tyrosine hydroxylase expression but not aromatic amino acid decarboxylase or GABA immunoreactivity. Neuroscience 36, 761-771 (1990).

24. Stone, D. M., Wessel, T., Joh, T. H. \& Baker, H. Decrease in tyrosine hydroxylase, but not aromatic L-amino acid decarboxylase, messenger RNA in rat olfactory bulb following neonatal, unilateral odor deprivation. Brain Res Mol Brain Res $\mathbf{8}$, 291-300 (1990).

25. Vassar, R. et al. Topographic organization of sensory projections to the olfactory bulb. Cell 79, 981-991 (1994).

26. Ressler, K. J., Sullivan, S. L. \& Buck, L. B. A zonal organization of odorant receptor gene expression in the olfactory epithelium. Cell 73, 597-609 (1993).

27. Morgan, K. T. \& Monticello, T. M. Airflow, gas deposition, and lesion distribution in the nasal passages. Environ Health Perspect 85, 209-218 (1990).

28. Young-Pearse, T. L., Chen, A. C., Chang, R., Marquez, C. \& Selkoe, D. J. Secreted APP regulates the function of full-length APP in neurite outgrowth through interaction with integrin betal. Neural Dev 3, 15 (2008).

29. Young-Pearse, T. L., Suth, S., Luth, E. S., Sawa, A. \& Selkoe, D. J. Biochemical and functional interaction of disrupted-in-schizophrenia 1 and amyloid precursor protein regulates neuronal migration during mammalian cortical development. J Neurosci 30, 10431-10440 (2010).

30. Wang, Z. et al. Presynaptic and postsynaptic interaction of the amyloid precursor protein promotes peripheral and central synaptogenesis. J Neurosci 29 10788-10801 (2009).
31. Hasegawa, S. et al. The protocadherin-alpha family is involved in axonal coalescence of olfactory sensory neurons into glomeruli of the olfactory bulb in mouse. Mol Cell Neurosci 38, 66-79 (2008).

32. Cutforth, T. et al. Axonal ephrin-As and odorant receptors: coordinate determination of the olfactory sensory map. Cell 114, 311-322 (2003).

33. Imai, T., Sakano, H. \& Vosshall, L. B. Topographic mapping--the olfactory system Cold Spring Harb Perspect Biol 2, a001776 (2010).

34. Serizawa, S. et al. A neuronal identity code for the odorant receptor-specific and activity-dependent axon sorting. Cell 127, 1057-1069 (2006).

35. Takeuchi, H. et al. Sequential arrival and graded secretion of Sema3F by olfactory neuron axons specify map topography at the bulb. Cell 141, 1056-1067 (2010).

36. Sakano, H. Neural map formation in the mouse olfactory system. Neuron 67 530-542 (2010)

37. Selkoe, D. J. Soluble oligomers of the amyloid beta-protein impair synaptic plasticity and behavior. Behav Brain Res 192, 106-113 (2008).

38. Hardy, J. The amyloid hypothesis for Alzheimer's disease: a critical reappraisal. J Neurochem 110, 1129-1134 (2009).

39. Ohno, M. et al. Temporal memory deficits in Alzheimer's mouse models: rescue by genetic deletion of BACE1. Eur J Neurosci 23, 251-260 (2006).

40. Ohno, M. et al. BACE1 gene deletion prevents neuron loss and memory deficits in 5XFAD APP/PS1 transgenic mice. Neurobiol Dis 26, 134-145 (2007)

41. Cole, S. L. \& Vassar, R. The Alzheimer's disease beta-secretase enzyme, BACE1. Mol Neurodegener 2, 22 (2007).

42. Vanderhaeghen, P. \& Cheng, H. J. Guidance molecules in axon pruning and cell death. Cold Spring Harb Perspect Biol 2, a001859.

43. Cai, H. et al. BACE1 is the major beta-secretase for generation of Abeta peptides by neurons. Nat Neurosci 4, 233-234 (2001).

44. Williams, E. O. et al. Delta protocadherin 10 is regulated by activity in the mouse main olfactory system. Frontiers in Neural Circuits $\mathbf{5}$

\section{Acknowledgements}

The authors thank E. Benz, Z. Doctor, A.C. Gomez, and P. Kim for excellent technical assistance, B. Hyman and B. Schrank and other members of the Albers lab for critical comments, and G. Barnea for antibodies to M71 and MOR28. This work was supported by the NIH (DP2-OD00662, awarded to M.W.A.) and an anonymous foundation.

\section{Author Contributions}

L.C., S.R. and M.W.A. designed experiments. L.C., G.T.R., S.R., and T.W.M. performed experiments, L.C., G.T.R., S.R., T.W.M. and M.W.A. analyzed data, M.W.A. wrote the main manuscript text. All authors reviewed the manuscript.

\section{Additional information}

Competing financial interests: The authors declare no competing financial interests.

License: This work is licensed under a Creative Commons

Attribution-NonCommercial-ShareAlike 3.0 Unported License. To view a copy of this license, visit http://creativecommons.org/licenses/by-nc-sa/3.0/

How to cite this article: Cao, L., Rickenbacher, G.T., Rodriguez, S., Moulia, T.W. \& Albers, M.W. The precision of axon targeting of mouse olfactory sensory neurons requires the BACE1 protease. Sci. Rep. 2, 231; DOI:10.1038/srep00231 (2012). 